Journal of Nuclear Medicine, published on October 30, 2020 as doi:10.2967/jnumed.120.251272

\title{
Dynamic ${ }^{68}$ Ga-PSMA-11 PET/CT for the primary evaluation of localized renal mass: a prospective study
}

Shay Golan ${ }^{1,2}$, Tzach Aviv ${ }^{1,2}$, David Groshar²,3, Maxim Yakimov²,4, Yaniv Zohar ${ }^{5}$ Yoad prokocimer ${ }^{1}$, Andrei Nadu ${ }^{1,2}$, Jack Baniel ${ }^{1,2},{ }^{*}$ Liran Domachevsky ${ }^{2,6}$ *Hanna Bernstine ${ }^{2,3}$

1Department of Urology Rabin Medical Center, Petach Tikva, Israel; ${ }^{2}$ Sackler Faculty of Medicine, Tel Aviv University, Tel Aviv, Israel; ${ }^{3}$ Department of Nuclear Medicine Rabin Medical Center, Petach Tikva, Israel; ${ }^{4}$ Department of Pathology, Rabin Medical Center, Petach Tikva, Israel; ${ }^{5}$ Department of Pathology, Rambam Health Care Campus, Haifa, Israel; ${ }^{6}$ Department of Nuclear Medicine, The Chaim Sheba Medical Center, Tel Hashomer, Israel

* LD and HB contributed equally to this manuscript as senior authors

Corresponding author: Shay Golan, M.D, Director of Urologic Oncology service Department of Urology, Rabin Medical Center Petach Tikva, Israel

Ph: $+972-3-9376554$

Email: shaygo1@gmail.com

Word count: 2636 


\section{Page 2 of 26}

Funding: This research did not receive any specific grant from funding agencies in the public, commercial, or not-for-profit sectors.

Running Title: Dynamic PSMA PET/CT for renal mass 


\title{
Page 3 of $\mathbf{2 6}$
}

\begin{abstract}
Purpose: The potential role of prostatic-specific membrane antigen (PSMA) positron emission tomography/computed tomography (PET/CT) in non-prostate cancer tumors has shown promising results. We examined the performance of dynamic ${ }^{68} \mathrm{Ga}-\mathrm{PSMA}-11$ PET/CT (DPSMA) for the evaluation of localized renal mass.
\end{abstract}

Methods: A prospective case series of patients with a newly diagnosed renal mass who were referred for surgery. DPSMA was performed in a standardized manner before surgery. The final surgical histology served as the standard of reference. PSMA expression in the tumor vasculature was assessed and staining intensity was scored. Tracer uptake and PSMA expression were compared between benign and malignant tissue.

Results: Of 29 enhancing renal masses evaluated in 27 patients, 24 (83\%) were malignant lesions. The median mean standardized uptake value (SUV mean $_{\text {) of }}$ benign and malignant lesions was 2.3 (IQR 2.2-2.7) and 6.8 (IQR 4.2-10.2), respectively $(p=0.009)$. Median $S U V_{\max }$ of benign and malignant lesions was 3.8 (IQR 3.3-4.5) and 9.4 (IQR 5.4-15.8), respectively ( $p=0.01)$, respectively. The median washout coefficient (K2) was significantly lower in malignant lesions compared to benign lesions $(0.16$ versus $0.80, p=0.006)$. Positive PSMA staining was found in 20/24 malignant lesions and 2/5 benign lesions $(p=0.04)$. 
Conclusions: This pilot study demonstrated DPSMA uptake and kinetics in localized renal masses. Increased ${ }^{68} \mathrm{Ga}-\mathrm{PSMA}-11$ tracer uptake and intra-tumoral retention correlate with PSMA expression in malignant renal tumors compared with benign renal masses, supporting further assessment of DPSMA as a potential tool for evaluating localized renal masses.

Keywords: kidney; carcinoma, ${ }^{68} \mathrm{Ga}-\mathrm{PSMA}-11, \mathrm{PET} / \mathrm{CT}$ 


\section{Page $\mathbf{5}$ of $\mathbf{2 6}$}

\section{INTRODUCTION}

The incidental detection of clinically localized renal masses continues to increase worldwide (1). While a substantial minority of renal masses are benign, the majority are malignant lesions with significant variability in biological aggressiveness. Although renal mass biopsy can discriminate fairly well between benign and malignant histology (2), it is an invasive procedure with a notable non-diagnostic rate (3). Therefore, imaging studies remain the mainstay diagnostic process.

The potential application of conventional imaging studies, such as computed tomography $(\mathrm{CT})$ and magnetic resonance imaging (MRI), to determine the histological nature of a renal mass has been thoroughly investigated. For example, T2-weighted MRI helps differentiate between lipid poor angiomyolipomas and clear cell renal cell carcinoma (ccRCC), while dynamic contrast-enhanced MRI can help differentiate between oncocytomas and chromophobe RCC (chRCC) (4,5). Diffusion-weighted MRI utilizes changes in tissue organization that affect the movement of water molecules. It has been investigated as a supplement to conventional MRI sequences for the prediction of renal malignancy (6). However, evidence suggests these modalities have an overall moderate accuracy for the prediction of malignancies and fair to moderate inter-reader agreement (7).

Positron emission tomography/computed tomography (PET/CT) provides essential information on lesions' morphological appearance and biological 


\section{Page $\mathbf{6}$ of $\mathbf{2 6}$}

behavior. Ongoing studies focus on optimizing the utilization of different radiotracers in RCC (8). Although prostatic-specific membrane antigen (PSMA) PET/CT was extensively investigated in prostate cancer, there is limited data regarding its uptake and localization/dynamics in other malignancies. PSMAassociated tracer uptake has been reported in a spectrum of benign and malignant lesions, including abdominal, thoracic, skeletal, and central nervous system (9).

PSMA is a glycoprotein with an internal transmembrane and external amino acid portion. As products of its folate hydrolase activity are associated with angiogenesis (10), the highly vascularized nature of RCC makes it a potential PSMA-avid tumor. Several small-scale studies investigated the use of PSMA PET in metastatic RCC patients with promising results (11). Here, we examined the role of dynamic ${ }^{68} \mathrm{Ga}-\mathrm{PSMA}-11 \mathrm{PET} / \mathrm{CT}$ (DPSMA) in the evaluation of localized renal mass.

\section{MATERIALS AND METHODS}

\section{Patients}

A prospective case series design was used. Patients referred for surgery at our institution with a newly diagnosed clinical stage I CT-enhanced renal mass were considered eligible for this study. DPSMA was performed up to three months before surgery. Surgical treatment (partial or radical nephrectomy) was administered independently of the PET/CT results. Data on patient characteristics and radiographic tumor features were collected. The study was 


\section{Page 7 of 26}

approved by the institutional review board and all patients signed an informed consent. ${ }^{68}$ GA-PSMA-11 PET was approved for clinical use by the Israel Ministry of Health in 2016.

\section{PET/CT Protocol}

Images were obtained from an integrated 8-slice PET-CT scanner (Discovery 710, GE Medical Systems, Milwaukee, WI) through dynamic acquisition initiated by injecting 2-4 mCi (75-150 MBq) of ${ }^{68} \mathrm{Ga}-\mathrm{HBED}-\mathrm{CC}-11$ PSMA ( $\left.{ }^{68} \mathrm{Ga}-\mathrm{PSMA}-11\right)$. After scout view of the pelvis, the study centered on the kidneys with PET coverage of $20.0 \mathrm{~cm}$ using a non-contrast low-dose (30 mA) CT scan. An automatic power injector (Dual-shot, Nemoto, Japan) inserted ${ }^{68} \mathrm{Ga}-$ PSMA-11 as a rapid bolus flushed with $50 \mathrm{~mL} 0.9 \%$ saline solution at 5.0 $\mathrm{mL} /$ second, resulting in a three-dimensional (3D) scan (matrix size 64x64, 3.27slice thickness) consisting of 18 sequential frames of 5 seconds each, followed by 9 frames of 60 seconds each. PET emission data after attenuation correction was reconstructed with a 3D ordered subset expectation maximization (OSEM) algorithm (two iterations, 20 subsets).

\section{Data Processing and Kinetic Analysis}

Blinded imaging analysis was performed with histology as the reference standard. All PET measurements and visual analyses were analyzed in consensus by a nuclear medicine physician (H.B.) and a radiology and nuclear medicine physician (L.D.), board-certified with 13 and 8 years of PET/CT experience, respectively. 


\section{Page 8 of 26}

The summation images used visual assessment in axial, sagittal, and coronal views. All ${ }^{68} \mathrm{Ga}$-PSMA-11-avid foci with higher uptake than adjacent renal parenchyma were considered visually suspicious for malignancy.

Dynamic PET data analysis was performed using the PMOD software (PMOD Technologies Ltd., Zurich, Switzerland). For dynamic PET/CT, a fused axial section at an anatomic level corresponding to known renal lesions was chosen, and a 3D volume of interest was created on the axial section of the fused PET/CT scan, then manually adjusted to encompass the maximum available lesion size in all three planes. Two additional volumes of interest were outlined for sampling the abdominal aorta and background activity, predefined as the liver parenchyma. Time activity curves were generated for the mean activities of each volume of interest, then fitted by a linear regression function.

The two-compartment pharmacokinetic model was used to simplify ${ }^{68} \mathrm{Ga}-$ PSMA-11 disposition in plasma and highly-perfused tissue, assuming instantaneous mixing in the bloodstream (12). Changes in ${ }^{68} \mathrm{Ga}-\mathrm{PSMA}-11$ plasma concentration are equivalent to changes in tissue concentrations; dosing, sampling, and elimination occur from the central compartment.

The following parameters were assessed as potential predictors of tumor histology: K1 - perfusion-related transfer coefficient from the plasma to the tumor $(\mathrm{mL} / \mathrm{cm} / \mathrm{min}), \mathrm{K} 2$ - rate constant of washout from the tumor to the plasma $(\mathrm{L} / \mathrm{min})$, mean and maximum standardized uptake value ( $S U V_{\text {mean }}$ and $\left.S U V_{\max }\right)$, and 


\section{Page 9 of 26}

tumor to background ratio (i.e. tumor $S U V_{\text {mean }} /$ iver tissue $S U V_{\text {mean; }}$ L:B). A threshold of 43 percent was used to calculate SUV mean.

\section{Histological Analysis}

All samples were evaluated using light microscopy and immunohistochemistry. Tumor type and subtype were determined by two senior pathologists (M.Y. and Y.Z.). The expression of PSMA on tumor vessels was evaluated by immunohistochemistry using two 4-mm tissue slides from formalinfixed, paraffin-embedded blocks of non-necrotic tumor areas. Immunostaining was performed using a monoclonal anti-PSMA antibody (NCL-L-PSMA; Novocastra, Leica Biosystems, Buffalo Grove, IL) at 1:100 dilution on an automated Ventana BenchMark XT slide stainer (Ventana Medical Systems, Inc., Oro Valley, AZ). The site (tumor neovasculature or tumor cell cytoplasm) and intensity of PSMA staining ("strong" [dark and diffuse], "moderate" [diffuse bright or focal intense], "weak" [bright and focal], or "absent") were determined. A prostatic adenocarcinoma slide was used as an internal control.

\section{Statistical Analysis}

Statistical analysis included descriptive analysis (median and interquartile range $[I Q R])$ for continuous and categorical variables, proportions for discrete variables, and comparative tests (Fisher's exact test for discrete variables; MannWhitney test for ordinal and continuous variables). All analyses were performed using Stata version 16.0 (Stata Corporation, College Station, TX). To evaluate the accuracy of $\mathrm{K} 1$ and $\mathrm{K} 2$ in determining the presence or lack of malignant 


\section{Page 10 of 26}

tumors, receiver operating characteristic (ROC) curves were drawn and the area under the curve (AUC) was calculated using MedCalc Statistical Software (version 19.4.0, Ostend, Belgium).

\section{RESULTS}

Twenty-seven consecutive patients diagnosed with 29 enhancing renal tumors between August 2018 and December 2019 were included in the analysis. The patients' median age was 66 years (IQR, 54-72) and 19/27 (70\%) were males. Two patients had bilateral synchronous tumors. Partial and radical nephrectomy were applied to 20/27 (74\%) and 5/27 (19\%) patients, respectively. Two patients underwent renal mass biopsy without consecutive surgery (one had oncocytoma and the other pursued active surveillance for low-grade ccRCC). Median time interval between DPSMA and surgery was 32 days (IQR 11-78). Most lesions $(24 / 29,83 \%)$ were malignant and the rest $(5 / 29,17 \%)$ were benign. Of malignant lesions, 18/29 (75\%) were ccRCC, 4/29 (17\%) were papillary (pRCC), and 2/29 (8\%) were chRCC. Most ccRCCs (14/18, 78\%) were low grade (World Health Organization/International Society of Urological Pathology [WHO ISUP] grade I/II), and 4 (22\%) were high grade (WHO ISUP III). Among the benign lesions, two were oncocytomas, two - lipid-poor angiomyolipomas, and one was a mixed epithelial and stromal tumor (MEST). Table 1 summarizes the patient and tumor characteristics.

\section{Association Between DPSMA and Clinico-Histological Features}




\section{Page 11 of 26}

${ }^{68}$ GA-PMSA-11 uptake was subjectively visualized in 17/29 (59\%) lesions with no significant difference between benign and malignant lesions ( $p=0.9$, Fig. 1). Median SUV mean of benign and malignant lesions was 2.3 (IQR 2.2-2.7) and 6.8 (IQR 4.2-10.2), respectively $(p=0.009)$. Median $S U V_{\max }$ of benign and malignant lesions was 3.8 (IQR 3.3-4.5) and 9.4 (IQR 5.4-15.8), respectively $(p=0.01)$. The $L: B$ ratio of benign and malignant lesions was $0.36(I Q R$ 0.34-0.52) and $1.18(I Q R 0.67-1.73)$, respectively $(p=0.01)$. The clinical stage was also associated with $S U V_{\text {mean }}(p=0.03)$ and marginally associated with $S U V_{\max }$ $(p=0.06)$. A comparison between benign and malignant renal masses is presented in Table 2.

The median washout coefficient, K2, was significantly lower in malignant compared to benign lesions $(0.18$ versus $0.7, p=0.02)$. No differences between malignant and benign lesions were found in the perfusion coefficient, $K 1(p=0.2)$. Figure 2 shows the ROC curves of K1 and K2. The AUC was $0.68(95 \% \mathrm{Cl}, 0.47-$ $0.85)$ for $\mathrm{K} 1$ and $0.83(95 \% \mathrm{Cl}, 0.64-0.95)$ for $\mathrm{K} 2$. Among malignant lesions, $\mathrm{K} 2$ was significantly lower in $\mathrm{ccRCC}$ and $\mathrm{pRCC}$ compared to chRCC (0.16 versus $1.4, p=0.02)$.

No differences were found between oncocytoma and RCC in their static parameters $S U V_{\text {mean }}$ and $S U V_{\max }(p=0.12$ and 0.18 , respectively), but the dynamic parameters, $\mathrm{K} 1$ and $\mathrm{K} 2$, were lower in RCC compared with oncocytoma. (K1: 0.4 [IQR 0.2-0.7] versus 1.2 [IQR 1-1.5], p=0.03; K2: 0.18 [IQR 0.1-0.24] versus 0.79 [IQR 0.71-0.88], p=0.03; Fig. 3). 


\section{Page 12 of 26}

\section{PSMA Immunohistochemistry}

Overall, PSMA expression was observed in 22/29 (76\%) specimens, with positive staining in $20 / 24$ malignant lesions, and $2 / 5$ benign lesions $(p=0.04)$. All four malignant lesions without staining were low-grade tumors; two ISUP grade 1 ccRCC, one chRCC, and one pRCC. The only benign lesions that showed PSMA expression were oncocytomas (Fig. 1).

While PSMA expression was noticed mostly in the endothelium of tumor vasculature, cytoplasmatic expression was observed in two pRCCs, one chRCC, and two oncocytomas. We also found differences in the associations between staining patterns and PSMA PET parameters: cytoplasmatic staining was associated with the washout coefficient K2 $(p=0.01)$ but not with the static parameters $(p=0.2$; Table 2$)$.

\section{DISCUSSION}

We investigated the potential role of DPSMA for the initial evaluation of localized renal mass. Our results demonstrate that in the majority of malignant renal tumors ${ }^{68} \mathrm{GA}-\mathrm{PSMA}-11$ uptake was increased with slower tracer washout compared with most benign lesions. This supports further assessment of DPSMA as a potential tool in evaluating localized renal masses.

Before ${ }^{68}$ GA-PSMA PET was available, immunohistochemical analyses confirmed the expression of PSMA in benign and malignant extraprostatic tissues (13). PSMA was detected in proximal tubules of the kidney and in the vasculature of several types of renal lesions (14). 


\section{Page 13 of 26}

The initial evaluation of ${ }^{68} \mathrm{GA}-\mathrm{PSMA} P E T$ for $\mathrm{RCC}$ was in the metastatic setting (13-15). In a prospective study of ten patients who underwent a systemic evaluation, ${ }^{68} \mathrm{GA}-\mathrm{PSMA}$ PET accurately detected metastases missed by conventional CT. Out of 35 biopsy-proved RCC sites testing true-positive on ${ }^{68} \mathrm{GA}-\mathrm{PSMA}$ PET, 11 were false-negative on CT. Information obtained by ${ }^{68} \mathrm{GA}-$ PSMA PET altered the management of two patients. Importantly, 8/10 patients had ccRCC, while two had pRCC and an unclassified tumor (15).

The reported detectability of non-clear cell RCC lesions on ${ }^{68} \mathrm{GA}-\mathrm{PSMA}$ PET was lower than that of ccRCC. An evaluation of 8 patients with metastatic non-clear cell RCC who underwent imaging with PSMA $\left[{ }^{18} \mathrm{~F}\right] \mathrm{DCFPyL}$ PET/CT, found that only $10 / 73(14 \%)$ metastatic lesions had a definitive radiotracer uptake above background. None of the lesions missed by conventional imaging were detected by PET (16). Similarly, in a retrospective cohort of eight patients with

oligometastatic RCC, ${ }^{68} \mathrm{Ga}$ PSMA-11 uptake was observed in seven patients with ccRCC but absent in one patient with pRCC (17).

Current literature regarding using ${ }^{68}$ GA-PSMA PET for evaluating localized renal mass is scarce, with data extrapolated from case series of metastatic patients (Table 3). Sawicki et al. described five primary RCC (3 ccRCC, one pRCC, and one chRCC) in a retrospective case series of six patients with metastatic disease (18). Although all primary tumors were PSMA avid, due to PSMA expression in the proximal tubules of the normal parenchyma, the tumor to background SUV $\max$ ratio was low. Thus the physiological PSMA uptake in the 


\section{Page 14 of 26}

kidneys limited the visualization of primary RCC, and the authors concluded that ${ }^{68}$ GA-PSMA PET does not have a role in the evaluation of primary renal masses. However, a comparison of ${ }^{68}$ GA-PSMA PET parameters between benign and malignant renal masses is not possible in a series of metastatic patients.

Although we did not find differences in visual tracer uptake $(p=0.9)$, significantly higher values of static ${ }^{68}$ GA-PSMA-11 PET parameters (SUV $\max$, $S U V_{\text {mean, }}$ and L:B SUV $V_{\text {mean }}$ ) were recorded in malignant compared to benign lesions. While angiomyolipomas and MEST showed no ${ }^{68}$ GA-PSMA-11 uptake and no PSMA staining, oncocytomas demonstrated substantial PSMA avidity. Furthermore, in line with previous reports $(16,17), \mathrm{pRCC}$ and $\mathrm{chRCC}$ showed lower static ${ }^{68} \mathrm{GA}-\mathrm{PSMA}-11$ PET values compared to ccRCC.

The differences observed in the kinetic parameters of ${ }^{68} \mathrm{GA}-\mathrm{PSMA}-11 \mathrm{PET}$ are important findings. The lower values of the washout coefficient (K2) in malignant lesions implies that ${ }^{68} \mathrm{GA}-\mathrm{PSMA}-11$ efflux is decreased in these lesions compared to benign lesions (Figs. 2 and 3). Interestingly, no differences were found between malignant and benign lesions in the transport coefficient (K1). In their investigation of dynamic ${ }^{18} \mathrm{~F}$-fluorodeoxyglucose (FDG) PET/CT, Nakajima et al. found no significant difference in early FDG accumulation between ccRCC and non-ccRCC, but a higher signal was observed in $c c R C C$ in the late phase (19). This may imply a faster tracer washout in benign lesions, as we observed. Even in a direct comparison between ccRCC and oncocytoma, we found lower $\mathrm{K} 2$ in the tumor tissue. 


\section{Page 15 of 26}

This diverse kinetics may be related to the heterogeneity of PSMA expression. While PSMA expression in the neovasculature of RCC is well documented, non-uniform cytoplasmatic tumoral staining has been reported. Some authors did not find cytoplasmatic PSMA staining in renal tumors $(13,14)$, whereas others found focal or strong staining $(20,21)$. We observed weak cytoplasmatic staining in some papillary and low-grade RCC and strong cytoplasmic staining in oncocytomas. In accordance with previous reports, the majority of RCC showed vascular endothelial staining (22). It is therefore possible that distinct staining patterns play a role in DPSMA kinetics. The statistically significant association found between cytoplasmatic staining and K2 supports this assumption.

Our study has several limitations. First, the small number of renal masses did not allow for sensitivity analyses, and potential cutoffs of DPSMA parameters could not be estimated. Second, the absolute number of analyzed benign masses was small. Nonetheless, the two most common benign renal masses are represented in this cohort and we provide preliminary dynamic PET and histological data that support the value of DPSMA in differentiating these masses from malignant renal masses. Third, observer-related bias was minimized by two highly experienced nuclear medicine physicians and two dedicated pathologists who reviewed each case independently and blindly. Fourth, because all patients were referred for surgery beforehand, selection bias is possible. Finally, while 


\section{Page 16 of 26}

dynamic PET/CT protocols are well established in the research setting, they are not widely adopted in routine clinical practice.

Despite these limitations, this is the first prospective evaluation of DPSMA for localized stage I renal mass. With technological advances, generating kinetic data may become a more accessible tool for clinicians. DPSMA may be a potential supplementary test to increase diagnostic confidence or an alternative for patients unable to undergo contrast CT due to renal insufficiency or severe allergic reaction to iodine.

The emergence of PSMA-based radioligand therapy in prostate cancer raises new potential applications for theranostics in other tumors as well (23). Upon binding to PSMA-expressing cancer cells, the radiolabeled compound is internalized and beta radiation induces cellular damage (24). However, radioligand distribution is a process that varies substantially between malignant and benign tumors, and among patients. The dynamic acquisition allows for a more robust measurement of radioligand kinetics and may, theoretically, assist in optimizing future treatments.

\section{CONCLUSION}

This is the first study to highlight ${ }^{68} \mathrm{Ga}-\mathrm{PSMA}-11$ kinetics in localized renal masses. We observed increased tracer uptake and slower washout in malignant renal masses compared to benign ones. These observations were further supported by distinct PSMA staining patterns in these tissues. Our findings 


\section{Page 17 of 26}

suggest that DPSMA has a potential role in the evaluation of renal masses and support further assessments in a larger patient cohort.

\section{DISCLOSURE}

\section{Funding}

This research did not receive any specific grant from funding agencies in the public, commercial, or not-for-profit sectors.

\section{Conflicts of Interest}

The authors declare they have no conflicts of interest.

\section{KEY POINTS}

Question: Does dynamic ${ }^{68} \mathrm{Ga}-\mathrm{PSMA}-11 \mathrm{PET}$ have the potential to serve as a diagnostic tool in the evaluation of localized renal mass?

Pertinent Findings: In this prospective case series of patients with newly diagnosed renal masses, we found statistically significant differences in dynamic

${ }^{68} \mathrm{Ga}-\mathrm{PSMA}-11$ PET parameters between benign and malignant lesions. This was further supported by differences in PSMA staining patterns in the final surgical specimen.

Implications for Patient Care: Dynamic ${ }^{68}$ Ga-PSMA-11 PET may potentially be a supplementary test to increase diagnostic confidence of localized renal mass or an alternative for patients unable to undergo contrast CT. 


\section{REFERENCES}

1. Znaor A, Lortet-Tieulent J, Laversanne M, Jemal A, Bray F. International variations and trends in renal cell carcinoma incidence and mortality. Eur Urol. 2015;67:519-530.

2. Volpe A, Finelli A, Gill IS, et al. Rationale for percutaneous biopsy and histologic characterisation of renal tumours. Eur Urol. 2012;62:491504.

3. Richard PO, Martin L, Lavallee LT, et al. Identifying the use and barriers to the adoption of renal tumour biopsy in the management of small renal masses. Can Urol Assoc J. 2018.

4. Hindman N, Ngo L, Genega EM, et al. Angiomyolipoma with minimal fat: can it be differentiated from clear cell renal cell carcinoma by using standard MR techniques? Radiology. 2012;265:468-477.

5. Cornelis F, Tricaud E, Lasserre AS, et al. Routinely performed multiparametric magnetic resonance imaging helps to differentiate common subtypes of renal tumours. Eur Radiol. 2014;24:1068-1080.

6. Kang SK, Zhang A, Pandharipande PV, Chandarana H, Braithwaite RS, Littenberg B. DWI for Renal Mass Characterization: Systematic Review and Meta-Analysis of Diagnostic Test Performance. AJR Am J Roentgenol. 2015;205:317-324.

7. Kay FU, Canvasser NE, Xi Y, et al. Diagnostic Performance and Interreader Agreement of a Standardized MR Imaging Approach in the Prediction of Small Renal Mass Histology. Radiology. 2018;287:543-553.

8. Meyer AR, Allaf ME, Rowe SP, Gorin MA. The role of molecular imaging in the characterization of renal masses. Curr Opin Urol. 2018;28:159-165.

9. de Galiza Barbosa F, Queiroz MA, Nunes RF, et al. Nonprostatic diseases on PSMA PET imaging: a spectrum of benign and malignant findings. Cancer Imaging. 2020;20:23. 
10. Carter RE, Feldman AR, Coyle JT. Prostate-specific membrane antigen is a hydrolase with substrate and pharmacologic characteristics of a neuropeptidase. Proc Natl Acad Sci U S A. 1996;93:749-753.

11. Ahn T, Roberts MJ, Abduljabar A, et al. A Review of ProstateSpecific Membrane Antigen (PSMA) Positron Emission Tomography (PET) in Renal Cell Carcinoma (RCC). Mol Imaging Biol. 2019.

12. Watabe $\mathrm{H}$, Ikoma $\mathrm{Y}$, Kimura $\mathrm{Y}$, Naganawa $\mathrm{M}$, Shidahara M. PET kinetic analysis--compartmental model. Ann Nucl Med. 2006;20:583-588.

13. Silver DA, Pellicer I, Fair WR, Heston WD, Cordon-Cardo C. Prostate-specific membrane antigen expression in normal and malignant human tissues. Clin Cancer Res. 1997;3:81-85.

14. Baccala A, Sercia L, Li J, Heston W, Zhou M. Expression of prostate-specific membrane antigen in tumor-associated neovasculature of renal neoplasms. Urology. 2007;70:385-390.

15. Rhee $\mathrm{H}$, Blazak J, Tham CM, et al. Pilot study: use of gallium-68 PSMA PET for detection of metastatic lesions in patients with renal tumour. EJNMMI Res. 2016;6:76.

16. Yin $Y$, Campbell SP, Markowski MC, et al. Inconsistent Detection of Sites of Metastatic Non-Clear Cell Renal Cell Carcinoma with PSMATargeted [(18)F]DCFPyL PET/CT. Mol Imaging Biol. 2018.

17. Siva S, Callahan J, Pryor D, Martin J, Lawrentschuk N, Hofman MS. Utility of (68) Ga prostate specific membrane antigen - positron emission tomography in diagnosis and response assessment of recurrent renal cell carcinoma. J Med Imaging Radiat Oncol. 2017;61:372-378.

18. Sawicki LM, Buchbender C, Boos J, et al. Diagnostic potential of $\mathrm{PET} / \mathrm{CT}$ using a (68)Ga-labelled prostate-specific membrane antigen ligand in whole-body staging of renal cell carcinoma: initial experience. Eur J Nucl Med Mol Imaging. 2017;444:102-107.

19. Nakajima R, Abe K, Kondo T, Tanabe K, Sakai S. Clinical role of early dynamic FDG-PET/CT for the evaluation of renal cell carcinoma. Eur Radiol. 2016;26:1852-1862. 
20. Dumas F, Gala JL, Berteau $P$, et al. Molecular expression of PSMA mRNA and protein in primary renal tumors. Int $J$ Cancer. 1999;80:799-803.

21. Spatz S, Tolkach $Y$, Jung $\mathrm{K}$, et al. Comprehensive Evaluation of Prostate Specific Membrane Antigen Expression in the Vasculature of Renal Tumors: Implications for Imaging Studies and Prognostic Role. J Urol. 2018;199:370-377.

22. Al-Ahmadie HA, Olgac S, Gregor PD, et al. Expression of prostatespecific membrane antigen in renal cortical tumors. Mod Pathol. 2008;21:727-732.

23. Weineisen M, Schottelius M, Simecek J, et al. 68Ga- and 177LuLabeled PSMA I\&T: Optimization of a PSMA-Targeted Theranostic Concept and First Proof-of-Concept Human Studies. J Nucl Med. 2015;56:1169-1176.

24. Yong KJ, Milenic DE, Baidoo KE, Brechbiel MW. Mechanisms of Cell Killing Response from Low Linear Energy Transfer (LET) Radiation Originating from (177)Lu Radioimmunotherapy Targeting Disseminated Intraperitoneal Tumor Xenografts. Int J Mol Sci. 2016;17. 


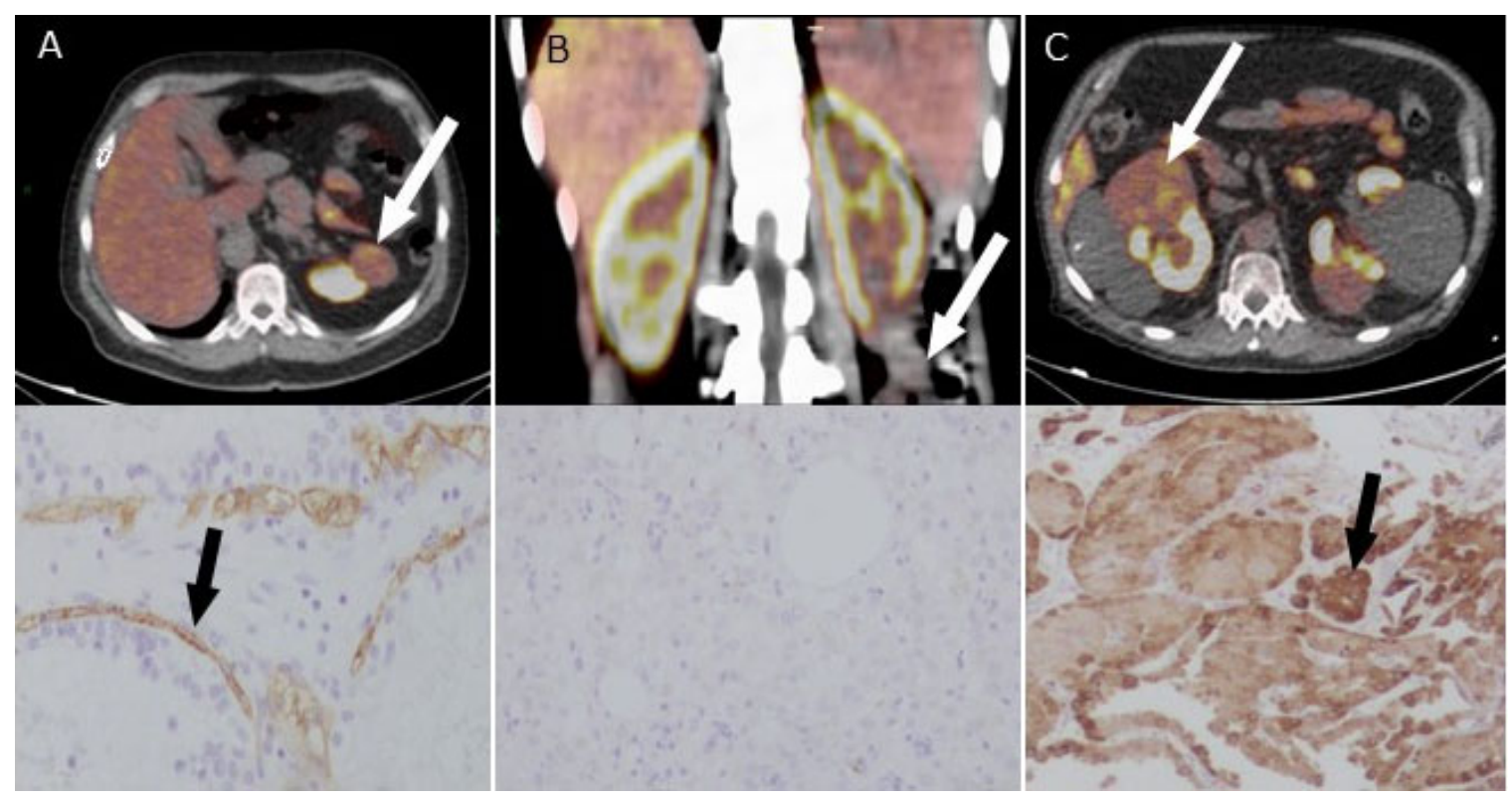

Figure 1. ${ }^{68} \mathrm{Ga}-\mathrm{HBED}-\mathrm{CC}-11-\mathrm{PSMA}$-fused PET/CT and the corresponding histopathological PSMA staining. (A) Tracer uptake and moderate renal neovasculature staining in WHO ISUP 2 ccRCC. (B) No uptake and no staining in lipid-poor angiomyolipoma. (C) Tracer uptake and strong cytoplasmatic staining in oncocytoma (white arrows= renal mass; black arrows= PSMA staining). Reduced from X200. 
A

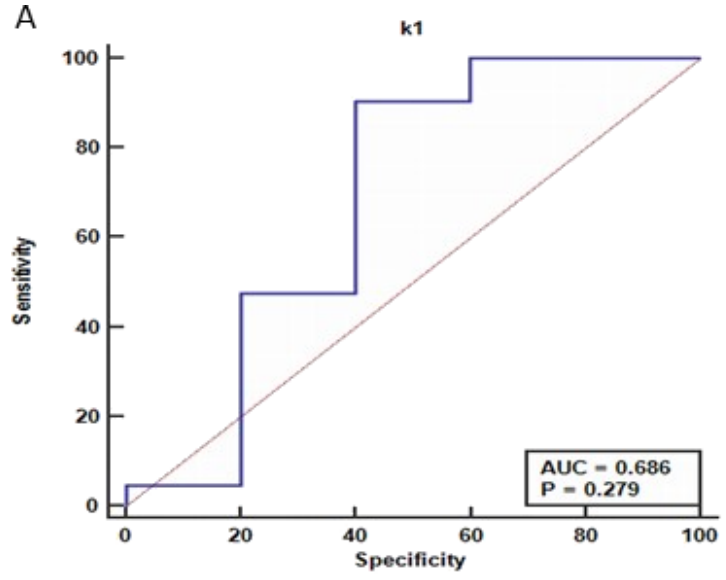

C
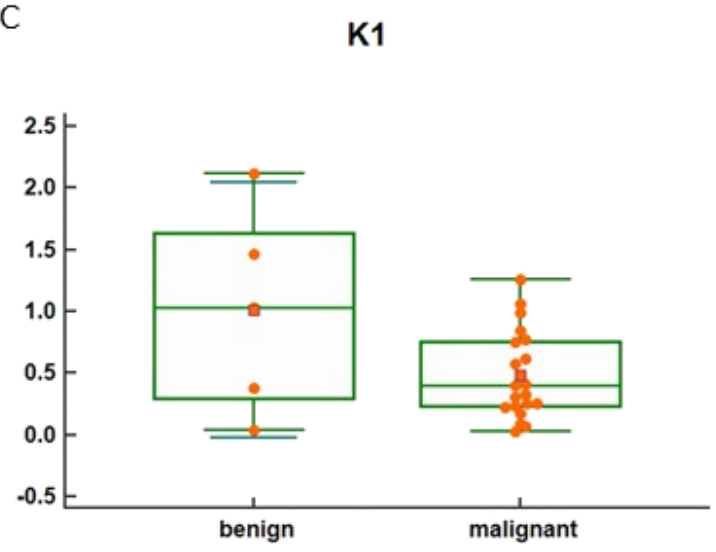

B

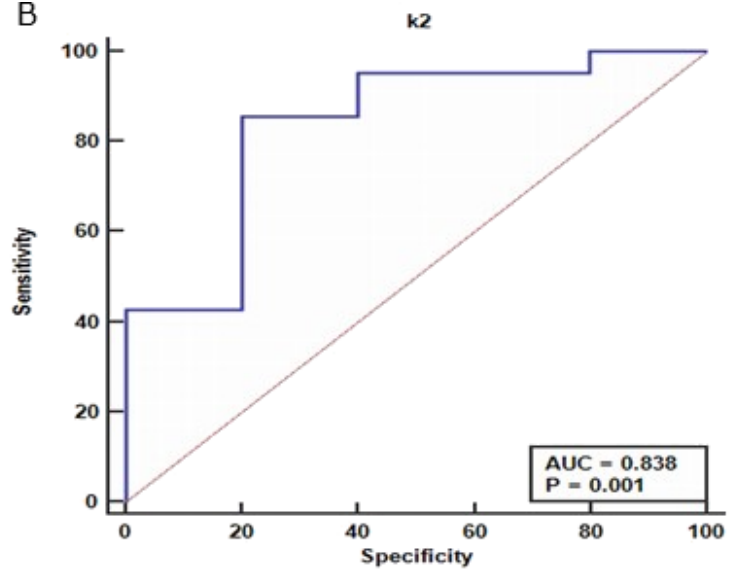

D

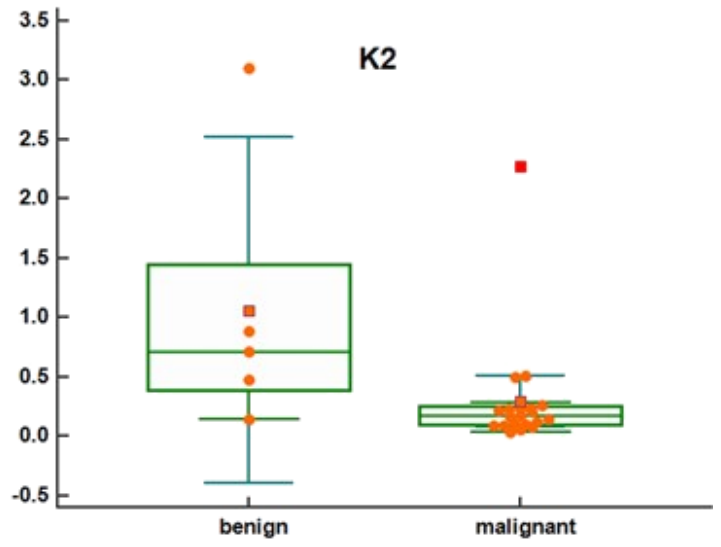

Figure 2. ROC curves and box plot of the perfusion coefficient $K 1(A, C)$ and the washout coefficient K2 $(B, D)$ as predictors of benign versus malignant histology. 


\section{Page 23 of 26}
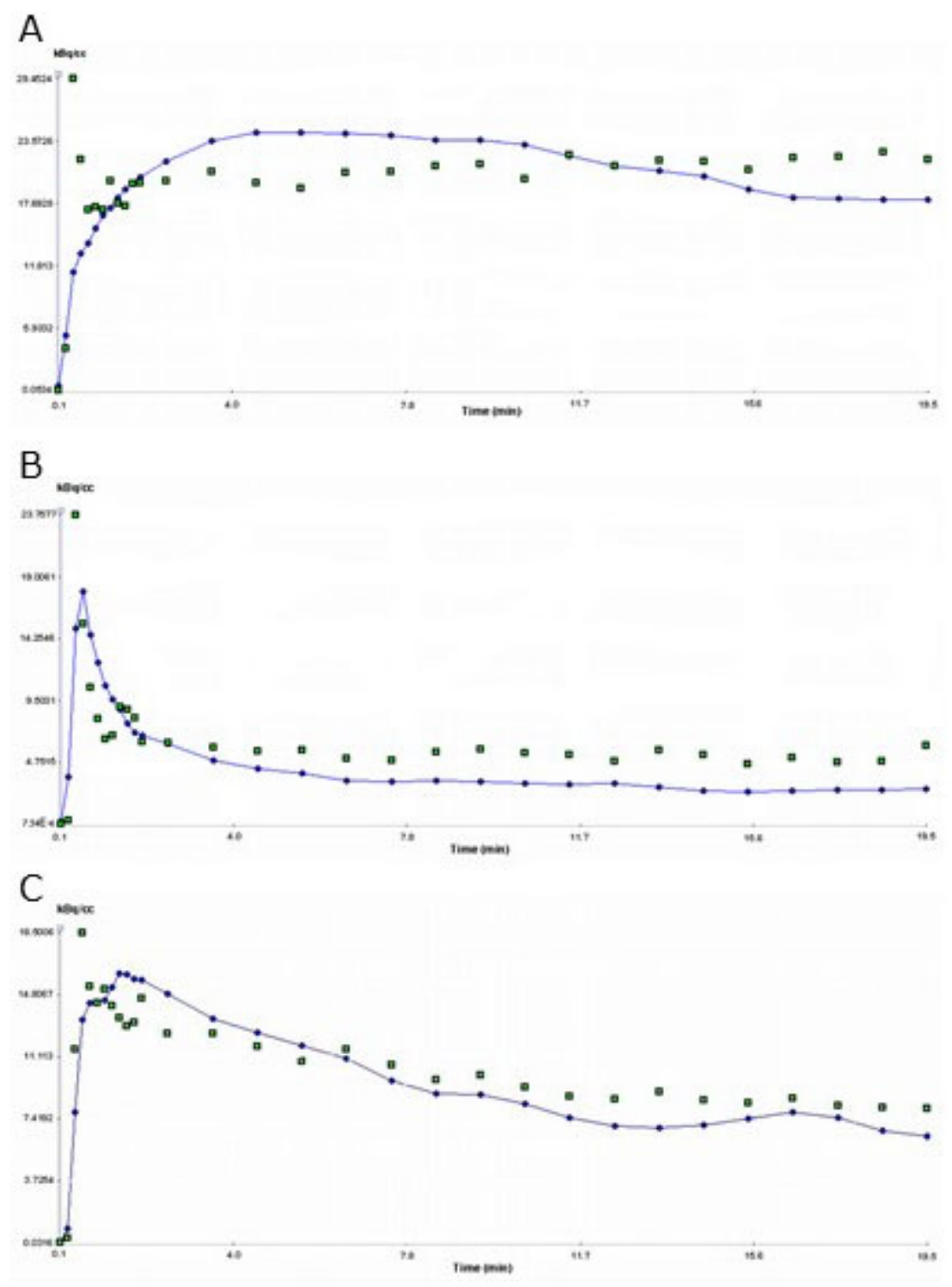

Figure 3. Linear fitted time-activity curves of ${ }^{68} \mathrm{Ga}-\mathrm{HBED}-\mathrm{CC}-11 \mathrm{PSMA}$ dynamic PET/CT for localized renal mass in the same patients as in Fig 1. (A) WHO ISUP $2 \operatorname{ccRCC}(\mathrm{K} 1=0.47, \mathrm{~K} 2=0.14)$. (B) lipid poor angiomyolipoma $(\mathrm{K} 1=2.12, \mathrm{~K} 2=3.1)$. (C) oncocytoma $(\mathrm{K} 1=1.03, \mathrm{~K} 2=0.88)$. (green dots= real activity at the determined time; blue line= gamma fit of the curve). 
Table 1. Demographics and clinical characteristics of 27 patients with 29 renal masses

\begin{tabular}{lc}
\hline Parameter\# & Value\# \\
\hline Age (years), median (IQR) & $66(54-72)$ \\
Male gender, n (\%) & $19(70)$ \\
BMI $\left(\mathrm{kg} / \mathrm{m}^{2}\right)$, median (IQR) & $27(24-30) \#$ \\
Charlson comorbidity index, median (IQR) & $4(3-6)$ \\
Tumor side, right (\%) & $15(52) \#$ \\
Tumor maximal diameter (cm), median (IQR) & $3.7(2.7-4.8) \#$ \\
Clinical Stage, n (\%) & \\
la & $18(62)$ \\
Ib & $11(38)$ \\
Pathology, n (\%) & $\#$ \\
Clear cell RCC & $18(62)$ \\
Papillary RCC & $4(14) \#$ \\
Chromophobe RCC & $2(7) \#$ \\
Oncocytoma & $2(7) \#$ \\
angiomyolipoma ( & $2(7) \#$ \\
Mixed epithelial and stromal tumor & $1(3)$ \\
(MEST) & \\
\hline
\end{tabular}


Table 2. Dynamic ${ }^{68}$ GA-PSMA-11 PET parameters and PSMA immunohistochemistry of benign versus malignant renal masses

\begin{tabular}{|c|c|c|c|}
\hline \# & $\begin{array}{l}\text { Benign masses } \\
\qquad(n=5) \#\end{array}$ & $\begin{array}{c}\text { Malignant } \\
\text { masses }(n=24)\end{array}$ & $P$ value \\
\hline PET PSMA & \# & & \\
\hline Visually positive lesions, $\mathrm{n}(\%)$ & $2(40) \#$ & $15(62)$ & 0.94 \\
\hline Lesion SUV mean, median (IQR) & $2.3(2.2-2.7) \#$ & $6.8(4.2-10.1)$ & 0.009 \\
\hline Lesion SUVmax, median (IQR) & $3.8(3.3 .-4.5)$ & $9.4(5.4-15.8)$ & 0.015 \\
\hline L:B SUV ${ }_{\text {mean, }}$ median (IQR) & $0.36(0.34-0.52) \#$ & $1.2(0.67-1.73)$ & 0.01 \\
\hline $\begin{array}{l}\text { Perfusion constant }-\mathrm{K} 1 \text {, } \\
(\mathrm{mL} / \mathrm{cm} / \mathrm{min}) \text {, median (IQR) }\end{array}$ & $1(0.38-1.5) \#$ & $0.4(0.24-0.75)$ & 0.2 \\
\hline $\begin{array}{l}\text { Washout constant -K2, } \\
\text { (L/min), median (IQR) }\end{array}$ & $0.7(0.47-0.88) \#$ & $0.18(0.1-0.24)$ & $0.02 \#$ \\
\hline $\begin{array}{l}\text { PSMA } \\
\text { immunohistochemistry }\end{array}$ & \# & & \# \\
\hline Any PSMA staining, $\mathrm{n}(\%)$ & $2(40)$ & $20(83)$ & 0.04 \\
\hline $\begin{array}{l}\text { Vascular endothelial staining, } \\
\mathrm{n}(\%)\end{array}$ & 0\# & $17(71)$ & 0.004 \\
\hline Cytoplasmatic staining, n (\%) & $2(40) \#$ & $7(29)$ & 0.6 \\
\hline Staining intensity ${ }^{1} \mathrm{n}(\%)$ & & & 0.65 \\
\hline Absent & $3(60)$ & $4(16)$ & \\
\hline Weak & 0 & $16(67)$ & \\
\hline Moderate & 0 & $3(13)$ & \\
\hline Strong & $2(40) \#$ & $1(4)$ & \\
\hline
\end{tabular}

1PSMA staining intensity was defined as "absent" (no staining); "weak" (bright and focal); "moderate" (diffuse bright or focal intense); and "strong" (dark and diffuse).

Note. Significant associations are highlighted in bold. IQR- interquartile range; BMI - body mass index; PSMA - prostatic-specific membrane antigen; SUV standard uptake value; L:B - lesion to background 
Table 3. Summary of literature utilization of PSMA PET for evaluation of primary renal lesions

\begin{tabular}{|c|c|c|c|c|c|c|}
\hline $\begin{array}{l}\text { Author } \\
\text { (year) }\end{array}$ & $\begin{array}{c}\text { Number } \\
\text { primary } \\
\text { renal lesions }\end{array}$ & Study design & Radiotracer & Histology & $\begin{array}{c}\text { Average SUV } \text { max }_{\text {of }} \\
\text { primary tumor }\end{array}$ & $\begin{array}{c}\text { Average lesion } \\
\text { to background } \\
\text { SUV } \\
\text { max }\end{array}$ \\
\hline $\begin{array}{l}\text { Sawicki et } \\
\text { al. (2016) }\end{array}$ & 5 & Retrospective & ${ }^{68} \mathrm{Ga}-\mathrm{PSMA}$ & $\begin{array}{l}3 \text { ccRCC, } 1 \\
\text { pRCC, } 1 \text { cRCC }\end{array}$ & $\begin{array}{c}9.9 \pm 9.2 \\
\text { (range 1.7-27.2) }\end{array}$ & $\begin{array}{c}0.2 \pm 0.3 \text { (range } \\
0.02-0.7 \text { ) }\end{array}$ \\
\hline $\begin{array}{l}\text { Siva et al. } \\
(2017)\end{array}$ & 8 & Retrospective & $\begin{array}{c}{ }^{68} \text { Ga-PSMA-11 } \\
\text { (HBED-CC) }\end{array}$ & $\begin{array}{l}7 \text { ccRCC , } 1 \\
\text { pRCC } \\
18 \text { ccRCC, } 4\end{array}$ & $\begin{array}{c}8.6 \\
\text { (range 0-26.5) }\end{array}$ & N/A \\
\hline
\end{tabular}

